\title{
Réforme catholique et modifications de l'espace sacré
}

Architecture et organisation de l'espace intérieur des édifices cultuels

\section{Bruno Restif}

\section{(2) OpenEdition}

\section{Journals}

Édition électronique

URL : http://journals.openedition.org/abpo/1341

DOI : 10.4000/abpo. 1341

ISBN : 978-2-7535-1492-8

ISSN : 2108-6443

Éditeur

Presses universitaires de Rennes

Édition imprimée

Date de publication : 20 décembre 2003

Pagination : 107-114

ISBN : 978-2-86847-933-4

ISSN : 0399-0826

Référence électronique

Bruno Restif, «Réforme catholique et modifications de l'espace sacré », Annales de Bretagne et des Pays de l'Ouest [En ligne], 110-4 | 2003, mis en ligne le 20 décembre 2005, consulté le 02 mai 2019. URL : http://journals.openedition.org/abpo/1341 ; DOI : 10.4000/abpo.1341 


\title{
Réforme catholique et modifications de l'espace sacré Architecture et organisation de l'espace intérieur
des édifices cultuels
}

\author{
Bruno RESTIF \\ ATER d'Histoire moderne \\ CRHISCO, Université Rennes 2 Haute-Bretagne \\ Université de Bretagne occidentale, Brest
}

Lancées pour partie par Alphonse Dupront, les études historiques associant espace et sacré ont connu un véritable succès dans les années 1980, dans le cadre du développement de l'anthropologie religieuse ${ }^{1}$, succès illustré dans l'Ouest de la France par le colloque consacré à ce sujet à Rennes en $1982^{2}$. Les approches ont été et restent multiples. Les historiens se sont intéressés à la sacralisation de l'espace dans le cadre d'une région ou d'une paroisse, en utilisant différents indices. Il peut s'agir des pèlerinages, des processions ou de la répartition des chapelles, et je pense là tout particulièrement aux travaux de Georges Provost sur la Bretagne et de Philippe Martin sur la Lorraine ${ }^{3}$. Marie-Hélène Froeschlé-Chopard, quant à elle, s'est intéressée, dans le cadre provençal, aux contrastes entre d'une part " le monde du dehors ", à savoir le rapport entre le terroir et les lieux de culte, les pratiques associées à ces lieux et les titulatures des édifices cultuels, et d'autre part " le monde du dedans ", celui du bâtiment consacré au culte ${ }^{4}$. Il s'agit alors d'étudier la place des autels et leurs titulatures, voire de s'intéresser aux tableaux figurant dans l'église et, à

1. Je reprends ici l'expression utilisée par Alphonse Dupront (DuPRonT, Alphonse, "Anthropologie religieuse ", LE GofF, Jacques et NORA, Pierre, Faire de l'histoire, t. 2 : Nouvelles approches, Paris, Gallimard, 1974, p. 142-183).

2. L'Espace et le sacré, Actes du colloque de Rennes, 1982, $\mathrm{n}^{\circ}$ spécial des Annales de Bretagne et des pays de l'Ouest, t. $90,1983, \mathrm{n}^{\circ} 2$.

3. Provost, Georges, La Fête et le sacré. Pardons et pèlerinages en Bretagne aux $17^{e}$ et $18^{e}$ siècles, Paris, Cerf, 1998, 530 p.; Martin, Philippe, Les Chemins du sacré. Paroisses, processions, pèlerinages en Lorraine du $16^{e}$ au $19^{e}$ siècle, Metz, Serpenoise, $1995,358 \mathrm{p}$

4. FroEsCHLÉ-ChOPARD, Marie-Hélène, Espace et sacré en Provence (16e-20 siècle). Cultes, images, confréries, Paris, Cerf, 1994, 605 p. 
l'exemple de Bernard Cousin, de tirer parti de la sémiologie de l'image pour étudier la répartition des éléments sur une surface peinte ${ }^{5}$.

D'autres auteurs, essentiellement des médiévistes et des historiens de l'art spécialistes du Moyen Âge, se sont intéressés à l'architecture des bâtiments cultuels afin de montrer les rapports qui existent entre celle-ci et un environnement culturel plus large. Il convient dans ce domaine de citer les précurseurs qu'ont été Carol Heitz, qui a travaillé sur les églises carolingiennes, et Erwin Panofsky, qui s'est attaché aux rapports entre l'architecture gothique et la théologie scolastique ${ }^{6}$. Dans le cadre d'un récent regain d'intérêt pour la liturgie, des théologiens, tel Louis Bouyer, et des chercheurs travaillant à la jonction entre l'histoire et l'histoire de l'art, comme Bernard Chedozeau, se sont attachés à étudier l'association entre architecture et liturgie?

L'architecture et l'organisation de l'espace intérieur des bâtiments cultuels, ainsi que celui de l'enclos dans lequel ils s'insèrent, connaissent, surtout au $17^{\mathrm{e}}$ siècle, de profondes modifications liées à la Réforme catholique. Il me semble intéressant d'étudier ces changements dans les églises paroissiales, afin de mieux les mettre en rapport avec la politique réformatrice menée à destination des laïcs. La Haute-Bretagne me paraît constituer une région se prêtant assez bien à cette étude, du fait à la fois du nombre et de la richesse des comptes de fabrique conservés, et du nouveau regard qui est porté depuis quelques années sur ses églises, longtemps un peu oubliées ou du moins négligées, et aujourd'hui redécouvertes grâce notamment aux travaux de Roger $\mathrm{Blot}^{8}$. En prenant appui sur trois diocèses haut-bretons, ceux de Rennes, Dol et Saint-Malo, qui comprennent au total 452 paroisses et 33 succursales nommées trèves ${ }^{9}$, et en croisant sources écrites et sources archéologiques (au sens large du terme), il convient d'envisager comment se manifeste le renforcement des séparations entre sacré et profane, ainsi que le souci de visibilité du chœur et notamment du maître-autel, enfin les changements induits dans le domaine des dévotions du fait de ces modifications spatiales.

5. Ibid.; FROESCHLÉ-CHOPARD, Marie-Hélène, La Religion populaire en Provence orientale au 18 siècle, Paris, Beauchesne, 1980, 418 p.; Cousin, Bernard, Le Miracle et le quotidien. Les ex-voto provençaux. Images d'une société, Aix-en-Provence, Éd. « Sociétés, Mentalités, Cultures", 1983, $339 \mathrm{p}$.

6. HEITZ, Carol, Recherches sur les rapports entre architecture et liturgie à l'époque carolingienne, Paris, SEVPEN, 1963, 286 p.; HEITZ, Carol, L'Architecture religieuse carolingienne : les formes et leurs fonctions, Paris, Picard, 1980, 288 p.; PANOFSKY, Erwin, Architecture gothique et pensée scolastique, Paris, Éditions de Minuit, 1992, 211 p.

7. BOUYER, Louis, Architecture et liturgie, Paris, Cerf, 1991, 109 p.; ChEDOzEAu, Bernard, Chœur clos, chœur ouvert. De l'église médiévale à l'église tridentine (France, $17^{e}-18^{e}$ siècle), Paris, Cerf, 1998, $124 \mathrm{p}$.

8. De nombreuses monographies réalisées par Roger Blot sont parues depuis 1992 dans La vie diocésaine, devenue en janvier 2003 Église en Ille-et-Vilaine.

9 . Ces chiffres concernent le $17^{\mathrm{e}}$ siècle. Le diocèse de Dol comprend 71 paroisses, en ne comptant pas les enclaves situées dans les diocèses autres que ceux de Rennes et de Saint-Malo. Le diocèse de Rennes compte 219 paroisses et 10 trèves, et celui de SaintMalo 162 paroisses et 23 trèves. 


\section{Le renforcement des séparations entre sacré et profane}

Depuis quelques années, il est admis que le concile de Trente, et $a$ fortiori sa réception officielle, ne marquent pas le début de la Réforme catholique, qui commence de façon progressive à partir de la fin du $15^{\mathrm{e}}$ siècle, avant de se déployer véritablement à partir du début du $17^{\mathrm{e}}$ siècle. Ce schéma, souligné depuis quelque temps par l'historiographie, trouve une illustration dans les évolutions architecturales visant à inscrire dans l'espace la séparation entre le sacré et le profane. Cela se traduit d'abord dans la délimitation de l'enclos, espace dans lequel se situe l'église et qui se trouve au contact de l'espace profane. Pour autant que nous puissions le saisir par le biais des comptes de fabrique, la construction d'un mur d'enclos semble dater presque partout du $15^{\mathrm{e}}$ siècle. Mais le mur ne ferme pas complètement l'espace, afin de permettre le passage des paroissiens, des convois mortuaires et des chariots transportant des matériaux pour les réparations de l'église. Les comptes de fabrique permettent de saisir l'installation, dans le courant du $16^{\mathrm{e}}$ siècle, d'échaliers, appelés " passees ", qui permettent aux paroissiens d'enjamber aisément le mur d'enceinte tout en interdisant l'accès aux animaux attirés aussi bien par l'herbe du cimetière que par les os qu'ils parviennent à déterrer. Quant à l'entrée principale, elle est de plus en plus souvent fermée par un portail constitué par un encadrement de pierre et de bois recouvert d'ardoise en son sommet, et de deux portes en bois généralement fermées à clé. Des portails de ce type sont par exemple réalisés à Landavran, près de Vitré, en 1533/1534, et à Gévezé, au nord de Rennes, en 1553/1554 ${ }^{10}$.

Au même moment, des ossuaires sont bâtis, presque toujours contre le mur sud de la nef, qui donne sur le cimetière par une porte dite " mortuaire ". L'ossuaire de Saint-Sulpice de Fougères est réalisé dès $1515^{11}$, et le diocèse de Rennes semble connaître une certaine avance chronologique dans leur réalisation. L'ossuaire, qui accueille les os des trépassés, une fois les corps décomposés, et est percé de jours afin de permettre aux fidèles de voir les ossements, vise peut-être à imposer un respect des dépouilles funèbres, et s'inscrit en tout cas dans cette volonté de séparation entre des espaces sacrés clos et des espaces profanes plus ouverts. Ce type de construction se répand au $17^{\mathrm{e}}$ siècle, sans cependant s'imposer partout puisqu'à la fin du $18^{\mathrm{e}}$ siècle des visites pastorales effectuées dans le diocèse de Rennes révèlent que certaines paroisses en sont encore dépourvues à cette date ${ }^{12}$. Un dépouillement quasi-exhaustif des documents d'archives, un inventaire non moins exhaustif des édifices conservés et la lecture des travaux des érudits de la fin du $19^{\mathrm{e}}$ et du début du $20^{\mathrm{e}}$ siècle - travail réalisé en collaboration avec Georges Provost - permettent d'affirmer que 77 ossuaires existent de façon certaine au milieu du $18^{\mathrm{e}}$ siècle

10. Arch. dép. d'Ille-et-Vilaine, 1 F 1609 et G Gévezé 5.

11. Arch. dép. d'Ille-et-Vilaine, G Fougères Saint-Sulpice 30.

12. Il s'agit des visites pastorales effectuées par $\mathrm{M}^{\mathrm{gr}}$ Bareau de Girac, évêque de Rennes dans les années 1770 (Arch. dép. d'Ille-et-Vilaine, G 3 B). 
dans le cadre des trois diocèses (en excluant les villes épiscopales), chiffre qui doit bien sûr être considéré comme un minimum. Plusieurs ossuaires constituent la partie supérieure d'un porche sud, élément apparu à la fin du $15^{\mathrm{e}}$ siècle, et qui se diffuse progressivement aux $16^{\mathrm{e}}$ et $17^{\mathrm{e}}$ siècles. Comportant des bancs situés en vis-à-vis, ce porche permet au conseil de paroisse de se réunir à l'extérieur de l'église proprement dite, toujours dans une optique de séparation entre l'espace sacré et les activités profanes.

À l'intérieur de l'église, d'autres soucis de séparation se font jour. Alors qu'à partir du $16^{\mathrm{e}}$ siècle les objets et vêtements liturgiques sont généralement placés dans la tour, souvent réalisée à cette époque, au $17^{\mathrm{e}}$ siècle les autorités ecclésiastiques souhaitent l'existence d'une pièce consacrée spécialement à cet effet ${ }^{13}$ : c'est la sacristie, qui ne communique qu'avec le chœur, et qui semble être construite dans la majorité des paroisses au $17^{\mathrm{e}}$ siècle. Les évêques se soucient également de faire poser des balustrades autour des fonts baptismaux et des autels, afin d'éviter que les fidèles n'y appuient leurs coudes ou n'y posent leur chapeau pendant la messe ${ }^{14}$.

Cette multiplication des clôtures correspond à un type de rationalisation des usages de l'espace : à chaque espace strictement délimité correspond une fonction, au caractère sacré plus ou moins affirmé, et il s'agit d'en contrôler les usages. Cette évolution s'accompagne, de façon apparemment paradoxale, d'un certain processus d'unification de l'espace.

\section{Le souci de visibilité du chœur, et notamment du maître-autel}

En effet, l'organisation de l'espace intérieur de l'église est totalement remise en cause au $17^{\mathrm{e}}$ siècle. Comme l'illustre le plan de l'église de Québriac (dans le diocèse de Saint-Malo), réalisé par Roger Blot ${ }^{15}$, les églises du début du $16^{\mathrm{e}}$ siècle sont généralement des églises d'un seul volume (fig. 1). Cet espace est d'une certaine manière unifié par la verrière axiale, orientée à l'est, vers Jérusalem et le soleil levant. Celui-ci illumine, chaque matin, cette grande verrière multicolore qui occupe l'essentiel du pignon d'abside, tandis que le prêtre célèbre la messe sur le maître-autel qui se trouve aux pieds de la maîtresse-vitre, prenant ainsi la tête de l'assemblée en marche, grâce au Sacrifice renouvelé, vers la Jérusalem céleste. Cette assemblée des fidèles n'a pas accès au chanceau, chœur liturgique réservé aux clercs, qui est séparé de la nef par un arc diaphragme. Cet arc est fermé par un chancel, cloison de bois ajourée, qui est appelé jubé lorsqu'il supporte une tribune. Au moins la moitié des églises paroissiales et

13. C'est ainsi qu'en 1620 l'évêque de Saint-Malo Guillaume Le Gouverneur exige, dans les statuts synodaux qu'il fait imprimer, " qu'es eglises esquelles n'y a point de sacristie, les paroissiens s'employent a en faire bastir une, et en icelle, un armoire, ou vestiaire, pour conserver les paremens et ornemens " (LE GOUVERNEUR, Guillaume, Statuts synodaux, Saint-Malo, Marcigay, 1620, 1122-79 p.).

14. Ibid.; THOREAU, Mathieu, Statuts et ordonnances de monseigneur l'illustrissime et reverendissime messire Mathieu Thoreau eveque et comte de Dol, Dol, Le Paigneux, 1678, 23 p.

15. Figure 1. BLot, Roger, "L'église de Québriac ", La vie diocésaine, 1993, n 8, p. 215. 
Figure 1 - L'église de Québriac au $16^{e}$ et au $17^{e}$ siècle

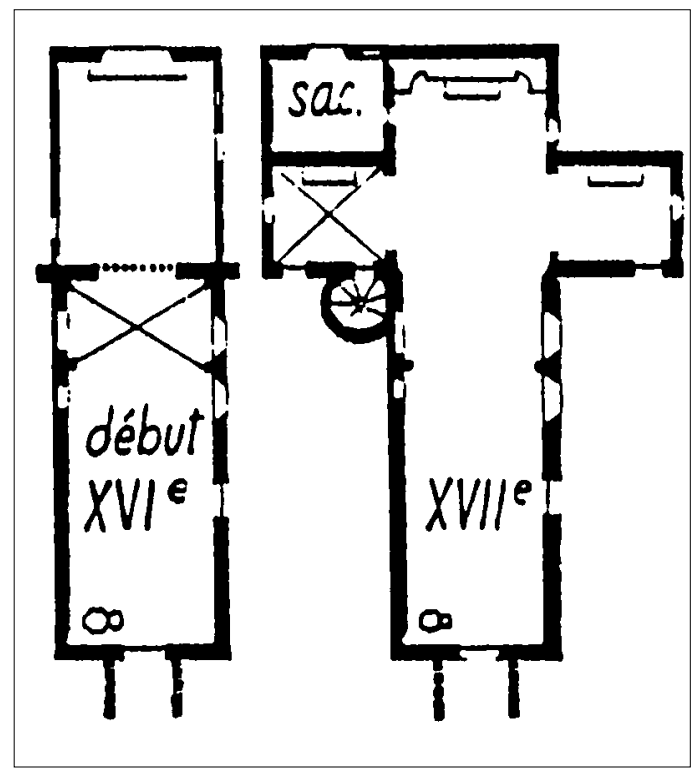

tréviales - chiffre fondé sur un dépouillement quasi-exhaustif des archives conservées et des vestiges archéologiques - sont refaites partiellement ou totalement entre le milieu du $15^{\mathrm{e}}$ et la fin du $16^{\mathrm{e}}$ siècle. Elles sont parfois agrandies d'une ou plusieurs chapelles, mais le principe qui préside à l'organisation de l'espace reste partout le même.

Au $17^{\mathrm{e}}$ siècle, d'après les sources disponibles, plus du tiers des églises paroissiales et tréviales font l'objet de modifications architecturales importantes ou non négligeables, alors même qu'une bonne partie d'entre elles ont été assez récemment refaites ou agrandies. C'est que, cette fois, l'organisation de l'espace est profondément modifiée. À Québriac comme dans bien des églises, l'arc diaphragme est détruit, et il en est de même du chancel ou jubé. Il y a donc en ce sens une unification de l'espace. D'autant que les autels secondaires qui étaient dans un certain nombre d'églises placés contre l'arc diaphgrame ou contre le chancel sont déménagés. En 1676, à Saint-Sulpice-la-Forêt, près de Rennes, le recteur propose de financer le déménagement " des deux autels de la nef de l'eglise, et de les restablir le longt des deux costés de l'eglise, atendu qu'ils ocupent le jour du grand autel, ce qui fait pour la gloire de Dieu et pour faciliter la veue du grand autel au peuples de sa paroisse ${ }^{16}$ ". L'objectif est bien en effet de rendre visible à tous le maître-autel et donc la célébration eucharistique. Le chancel (ou jubé) est remplacé par une clôture nettement plus basse, appelée " balustre ", qui est à hauteur d'appui et sert de table de communion. Éventuellement, la sacristie est surmontée d'une tribune qui donne directement

16. Arch. dép. d'llle-et-Vilaine, G 3 B. 
sur le chœur et est réservée aux chantres, en remplacement de la tribune du jubé, comme c'est le cas, à partir des années 1650-1660 à Chavagne, dans le diocèse de Rennes, et à Tréfumel, dans le diocèse de Saint-Malo ${ }^{17}$.

Dans un certain nombre d'églises, le maître-autel est surélevé à l'aide de gradins, afin d'en améliorer la visibilité. Mais celle-ci est gênée par la maîtresse-vitre, qui est aveuglante, et ne convient donc pas à cette nouvelle organisation de l'espace. Dans les années 1620, apparaissent dans les diocèses de Rennes et du Mans les premiers retables, structures architecturées comportant des représentations figurées et alors placées derrière le maître-autel afin de le mettre en valeur ${ }^{18}$. Le retable majeur bouche la maîtresse-vitre, et le premier cas certain d'installation de retable ayant entraîné la suppression de la verrière axiale se situe à Billé, près de Fougères, où le retable réalisé en 1629 par Jean Martinet monte à huit mètres de hauteur. Ces transformations nécessitent généralement un amé-

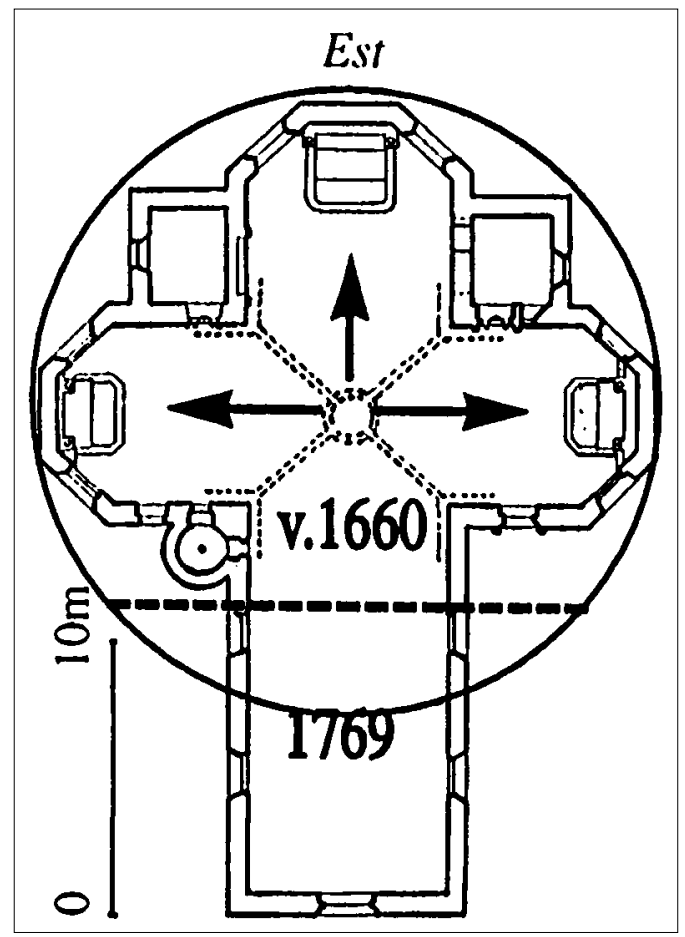

Figure 2 - La reconstruction du chour de l'église de Fleurigné vers 1660

17. Arch. dép. d'Ille-et-Vilaine, G Chavagne 4. À Tréfumel, la date 1660 est inscrite audessus de la porte de la sacristie.

18. BLot, Roger, « Premiers retables lavallois », La Vie diocésaine, 1996, n 5, p. 126-127, $\mathrm{n}^{\circ}$ 6, p. 150-151; MÉNARD, Michèle, Une Histoire des mentalités religieuses aux $17^{e}$ et $18^{e}$ siècles. Mille retables de l'ancien diocèse du Mans, Paris, Beauchesne, 1980, 467 p.; SALBERT, Jacques, Les Ateliers de retabliers lavallois aux $17^{e}$ et $18^{e}$ siècles : étude historique et artistique, Paris, Klincksieck, 1976, 540 p. 
nagement du chœur. À Québriac, le chœur est agrandi par la construction de deux chapelles formant transept, et le retable majeur est réalisé par Mathurin Thé en 1684. Presque partout, de nouvelles fenêtres sont percées afin de distribuer différemment la lumière. Il s'agit de fenêtres qui éclairent de façon latérale le retable et l'autel, et pour lesquelles le verre blanc semble souvent utilisé, permettant ainsi une bonne visibilité. Dans certaines églises, le chœur est entièrement refait. À Fleurigné, près de Fougères, grâce au financement assuré par les châtelains du Bois-Février, le chanceau prend vers 1660 la forme de trois chœurs à pans coupés presque parfaitement symétriques ${ }^{19}$. L'emplacement des fenêtres assure alors un très bon éclairage des autels et des retables (fig. 2).

Cette profonde modification de l'espace intérieur des églises, liée au souci de rendre visible le maître-autel et de le magnifier par la présence du retable, induit des changements dans les dévotions et les pratiques.

\section{Les changements induits dans le domaine des dévotions}

Au $16^{\text {e }}$ siècle, la maîtresse-vitre comprend presque toujours une représentation de la Passion, et le fait que le chancel constitue une cloison ajourée permet aux fidèles d'adorer le Corpus Christi lors de l'élévation. Cette insistance sur le sacrifice christique persiste évidemment au $17^{\mathrm{e}}$ siècle, mais se manifeste de façon différente, puisqu'il s'agit désormais de rendre visible la célébration eucharistique et la Présence réelle. Alors que les espèces consacrées étaient jusqu'alors conservées dans un sacraire, niche ménagée dans un mur du chœur et invisible aux fidèles, elles sont, au $17^{\mathrm{e}}$ siècle, placées dans un tabernacle, armoire de forme architecturale placée au-dessus du maître-autel et qui s'intègre au retable. La magnificence des tabernacles s'accroît au fil du siècle, et ils finissent par prendre la forme de somptueux édifices baroques en réduction.

Dans la nef sont placés deux meubles qui illustrent deux axes majeurs de la politique menée par l'Église de la Réforme catholique à l'égard des laïcs : il s'agit de la chaire pour la prédication et du confessionnal qui vise à permettre à la fois l'individualisation et l'intériorisation de la vie religieuse. La plus ancienne chaire conservée l'est à Rannée, dans le diocèse de Rennes, et date des dernières années du $16^{\mathrm{e}}$ siècle, tandis qu'à Louvignéde-Bais, dans le même diocèse, trois confessionnaux sont réalisés en $1614 / 1615^{20}$.

De nombreuses chapelles sont construites pour accueillir les dévotions alors en vogue et le financement de leur réalisation est parfois assuré par des confréries, comme c'est souvent le cas pour les chapelles consacrées au Rosaire. Ces chapelles accueillent à leur tour des retables, qui constituent de véritables systèmes iconographiques. Ils s'accompagnent du déve-

19. Figure 2. ВLот, Roger, "Église Saint-Martin de Fleurigné ", La vie diocésaine, 1997, $\mathrm{n}^{\circ} 13$, p. 343-346.

20. Arch. dép. d'Ille-et-Vilaine, G Louvigné-de-Bais 33. 
loppement de la peinture sur toile et de la réalisation de nouvelles statues, tandis que les anciennes semblent disparaître pour une part non négligeable d'entre elles. La majorité des tableaux et des statues qui se trouvent aujourd'hui sur les retables datent du $19^{\mathrm{e}}$ siècle, et rares sont ceux qui ont conservé leur programme. Il apparaît cependant que l'iconographie présente le plus souvent les personnes divines, la Vierge, sainte Anne et saint Joseph, les apôtres, saint Jean-Baptiste et les principaux saints protecteurs et guérisseurs (saint Sébastien, saint Roch, saint Fiacre), au détriment peutêtre de saints plus locaux qui n'apparaissent pas sur les retables.

Ainsi, les évolutions dans l'organisation de l'espace intérieur des églises (et de l'enclos) et les modifications architecturales constituent une des meilleures illustrations de la politique menée par l'Église de la Réforme catholique, et de ce fait fournissent un des indices les plus précieux pour suivre l'application de cette Réforme. Cet espace est à son tour modifié au $19^{\mathrm{e}}$ siècle puis dans la seconde moitié du $20^{\mathrm{e}}$ siècle, à tel point que sa logique a souvent été oubliée aujourd'hui, ce qui a des conséquences non négligeables lorsque des restaurations sont menées et aboutissent, par ignorance, à un résultat inverse de celui recherché initialement, comme à Longaulnay où la restauration de l'enclos s'est traduite par la destruction du porche, de l'ossuaire et de la croix ${ }^{21}$.

\section{RÉSUMÉ}

L'architecture et l'organisation de l'espace intérieur des édifices cultuels, ainsi que celui de l'enclos dans lequel ils s'insèrent, connaissent, surtout au $17^{\mathrm{e}}$ siècle, de profondes modifications liées à la Réforme catholique. Ces évolutions se caractérisent essentiellement par le renforcement des séparations entre sacré et profane, qui s'inscrit dans l'espace, et le souci de visibilité du chœur, et notamment du maître-autel. Bien évidemment, ces bouleversements de l'organisation spatiale induisent des changements dans le domaine des dévotions.

\section{ABSTRACT}

The architecture and arrangement of the cultual buildings' interior spaces as well as of their enclosures undergo deep changes related to the Catholic Reformation, namely during the 17th century. These evolutions are essentially caracterised by a more distinct separation in space between the Sacred and the Profane. Another distinguishing mark is the striving after a stronger visibility of the choir and, in particular, the high altar. Naturally, these changes on the spatial level have also an impact ondevotional practices.

21. Il est évident que ces travaux ont été menés avec la meilleure volonté. 